\title{
Collection-Mode Near-Field Imaging With 0.5-THz Pulses
}

\author{
Oleg Mitrofanov, Mark Lee, Julia W. P. Hsu, Igal Brener, Member, IEEE, Roey Harel, John F. Federici, \\ James D. Wynn, Loren N. Pfeiffer, and Ken W. West
}

\begin{abstract}
High spatial resolution imaging with terahertz pulses is implemented with a novel collection-mode near-field probe. The spatial resolution capabilities of the system are in the range of few micrometers. We demonstrate resolution of $7 \mu \mathrm{m}$ using $0.5-\mathrm{THz}$ pulses and discuss performance of the collection-mode near-field probes and image properties.
\end{abstract}

Index Terms-Near field, picosecond pulses, scanning microscopy, subwavelength aperture, terahertz pulses.

\section{INTRODUCTION}

I MAGING technology has progressed rapidly into the terahertz region of the electromagnetic spectrum during the last few years [1], owing to development of the terahertz timedomain (or terahertz time-resolved) spectroscopy (THz-TDS) technique [2], [3]. This method covers a wide spectral window from 0.1 to $40 \mathrm{THz}$. The system employs a small power in the terahertz beam, but it has exceptional sensitivity. This combination makes the THz-TDS system a powerful tool for far-infrared imaging [4]-[7].

The long wavelength of the radiation, however, limits spatial resolution of conventional terahertz imaging systems due to diffraction [1], [4]. Near-field scanning imaging techniques avoid this drawback and improve spatial resolution significantly. Various methods based on this approach have been demonstrated, pushing the resolution capabilities to several tens of micrometers [7]-[11]. Among them is a dynamic aperture approach that potentially can improve resolution to a few micrometers [11]. However, application of this method is limited to semiconductor surfaces and images are related to the concentration of photogenerated carriers. In this paper, we present an imaging method that provides very high spatial resolution. This scanning method is based on detection of the electric field of the terahertz pulses transmitted through an object using a probe with a subwavelength aperture.

The resolution capabilities of this method are considerably better than the wavelengths of the employed terahertz radiation $(120-1500 \mu \mathrm{m})$. Furthermore, the resolution is independent of the wavelength. The combination of the near-field microscopy

Manuscript received January 4, 2001; revised July 9, 2001.

O. Mitrofanov, M. Lee, J. W. P. Hsu, L. N. Pfeiffer, and K. W. West are with Bell Laboratories, Lucent Technologies, Murray Hill, NJ 07974 USA.

I. Brener and R. Harel are currently with Tellium, Inc., Oceanport, NJ 07757 USA.

J. F. Federici is with the New Jersey Institute of Technology, University Heights, Newark, NJ 07102 USA.

J. D. Wynn is currently with Princeton Optronics, Mercerville, NJ 08619 USA.

Publisher Item Identifier S 1077-260X(01)09932-4.

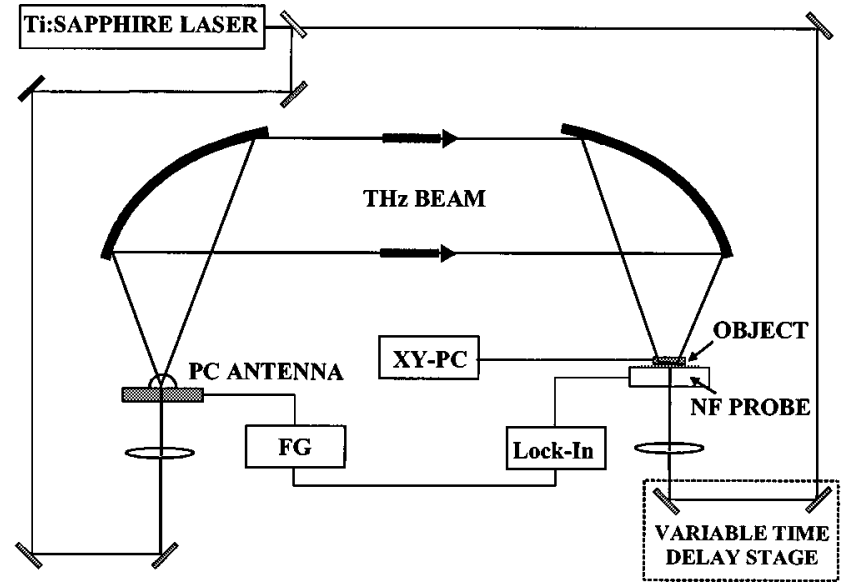

Fig. 1. Schematic diagram of the terahertz near-field imaging setup. XY-PC is the $x y$-position control equipment for scanning and FG is a function generator, which applies the alternating bias to the PC antenna.

scheme with the THz-TDS technique allows for study of the temporal evolution of the electromagnetic field in the near-field zone of objects. The broadband terahertz source allows spectroscopic applications on a micrometer scale.

A spatial resolution better than the wavelength can be achieved if the evanescent components of the field scattered by the object are detected. The evanescent field exists only within a short (compared to the wavelength) distance from the object. Detection of the evanescent field is possible by introducing an aperture-type probe into the near-field region. The local field in front of the aperture determines waves, which couple into the probe. These waves carry information about an area of the object, where the probe is placed. By scanning the object in front of the probe, one constructs a near-field image. Spatial resolution of this method is defined by the aperture size and is not limited by diffraction.

\section{EXPERIMENTAL SETUP}

The terahertz near-field imaging setup is presented in Fig. 1. Electromagnetic pulses with a central frequency of $0.5-0.6 \mathrm{THz}$ are generated by the transient current in a photoconducting (PC) switch excited by optical pulses from a mode-locked Ti-sapphire laser $\left(\lambda_{\mathrm{c}}=800 \mathrm{~nm}, \tau_{\mathrm{FWHM}}=100 \mathrm{fs}\right)$. The repetition rate of the laser system is $80 \mathrm{MHz}$. The terahertz beam is focused on the object through a transparent substrate by means of two off-axis parabolic mirrors. The beam waist in the object plane is $\sim 2 \mathrm{~mm}$ [full-width at half-maximum (FWHM)], which 


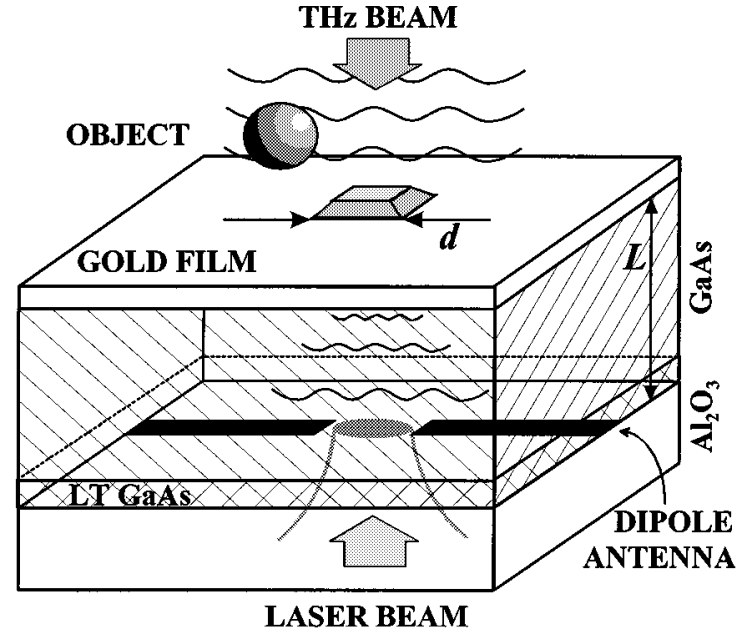

Fig. 2. Schematic diagram of the near-field probe.

is much larger than the objects used in the experiments. Therefore, the illumination can be considered uniform. The near-field probe is located behind the sample at a distance of $1-2 \mu \mathrm{m}$. The probe consists of a small aperture in a metallic screen and a PC antenna that detects terahertz pulses.

Generation of the terahertz pulses is slowly modulated (12 $\mathrm{kHz}$ ) by applying an alternating bias to the emitting PC switch. The detecting antenna in the probe is gated by the optical pulses from the same laser. Photocurrent in the antenna, induced by the incident terahertz field, is measured using a lock-in amplifier. A variable time-delay stage allows for the time-domain sampling of the terahertz pulses. An automated $x y$-translation stage scans an object in front of the probe. The near-field images are constructed either at a fixed time delay or in the time domain for every position $(x, y)$. The terahertz transducers, terahertz optics, and object are enclosed into a vapor tight box purged with nitrogen gas to reduce absorption and dispersion due to water vapor.

A schematic diagram of the near-field probe is presented in Fig. 2. A square shape entrance subwavelength aperture of size $d(5-50 \mu \mathrm{m})$ is lithographically defined on a surface of the probe in a 600-nm gold film evaporated on a GaAs layer. A GaAs protrusion through the aperture enhances field coupling into the probe. The PC planar antenna is embedded between the thin layer of GaAs $(3-10 \mu \mathrm{m})$ and a sapphire substrate. The antenna is fabricated on a $1-\mu \mathrm{m}$-thick low-temperature grown GaAs epilayer. The sapphire substrate supports the structure while allowing the optical pulses to gate the antenna from the substrate side. The details of the probe fabrication are described elsewhere [12].

\section{NEAR-FIELD PROBE}

\section{A. Probe Sensitivity}

Most of the incident terahertz power is reflected from the metallic screen, and coupling into the probe aperture is extremely weak [13]. Probe sensitivity, however, can be improved by efficient detection of the coupled terahertz field [12]. The electric field that exists behind the illuminated subwavelength

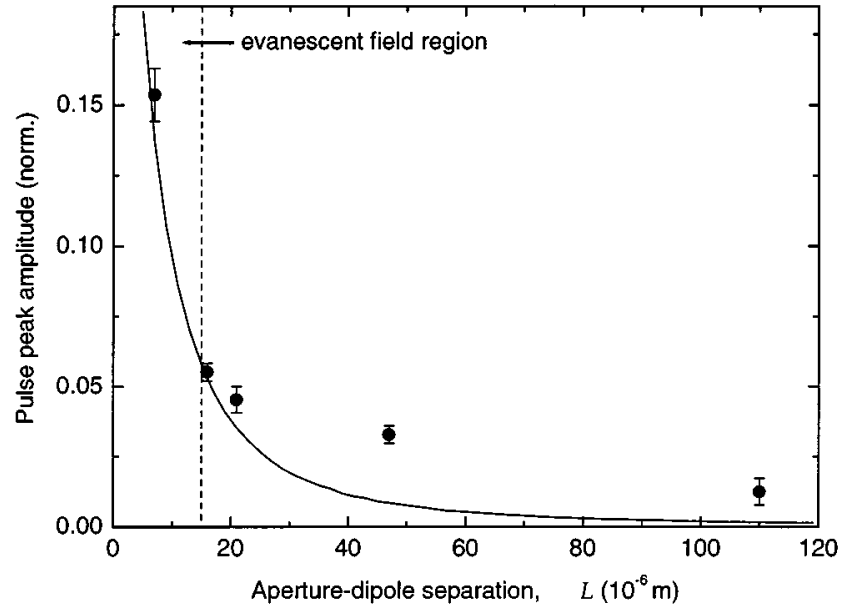

Fig. 3. Peak amplitude of the terahertz pulse as a function of the position of the detecting antenna for the $30 \times 30 \mu \mathrm{m}^{2}$ aperture probe (circles). The solid line shows the calculated amplitude of the evanescent components of a $\lambda=430 \mu \mathrm{m}$ wave incident on a $30-\mu$ m-diameter aperture.

aperture consists of modes with real and imaginary longitudinal $k_{z}$-vectors [14]. The latter are usually referred to as evanescent modes. The amplitude of the evanescent modes is significantly larger than that of the propagating modes (real $\left.k_{z}\right)$ at a short distance from the aperture $(z<d / 2)$ [15]. At a distance approximately equal to the aperture size, their contribution is comparable. The amplitude of both mode types decreases as distance $z$ increases, but decay is much more rapid in the case of the evanescent modes. Only the modes with real wavenumbers can transfer energy to the far-field zone $(z \gg \lambda)$. Consequently, the evanescent modes are not detected in conventional collection-mode near-field microscopy.

In our probe design, the detecting antenna is placed close to the aperture $(z \leq d / 2)$. Therefore, not only the propagating, but also the evanescent modes of the radiation coupled into the aperture radiation contribute to the measured filed. As a result, the sensitivity of the near-field probe improves.

The advantage of the electric field detection in the vicinity of the aperture is demonstrated by comparing the probes with different separation between the aperture and the detection point. The thickness of the GaAs layer in the probe determines the separation between the aperture and the detecting antenna. We fabricated probes with a $30 \times 30 \mu \mathrm{m}^{2}$ aperture and a GaAs layer ranging between 7 and $110 \mu \mathrm{m}$.

The terahertz beam is normally incident on the aperture plane. The on-axis amplitude of the electric field of the terahertz pulse is plotted as a function of the distance from the aperture in Fig. 3. The amplitude is normalized to that of the incident terahertz pulse. The electric field amplitude decreases very quickly as the detection point moves away from the aperture. The sensitivity of the probe with $L=7 \mu \mathrm{m}$ is an order of magnitude higher than that of the probe with $L=110 \mu \mathrm{m}$.

To evaluate the relative mode contribution to the signal at different distances $z$, we calculated the amplitude of the evanescent components of a $\lambda=430 \mu \mathrm{m}$ wave incident on a $30-\mu$ m-diameter aperture. The result is shown by the solid curve in Fig. 3. The curve is normalized to the amplitude of the incident field. The calculations, based on the modal 
approximation [15], distinguish waves with real and imaginary wavenumbers. The wavelength of $430 \mu \mathrm{m}$ corresponds to the spectral maximum of the terahertz field measured by the probe. Note that the space behind the aperture is filled with a high refractive index material that reduces the effective wavelength. The detecting antenna is located between GaAs $(n \sim 3.6)$ and $\mathrm{Al}_{2} \mathrm{O}_{3}(n \sim 3.1)$.

In spite of the single wavelength approximation, the calculated evanescent wave amplitude function agrees well with the experimental data at $z<d / 2$, where contribution of the evanescent modes is dominant. Starting at distance $z \sim 15 \mu \mathrm{m}$, which corresponds to $\sim d / 2$ in normalized units, the calculated amplitude of the evanescent modes becomes smaller than the measured amplitude. This fact confirms the diminishing contribution of the waves with imaginary $k_{z}$-vector at larger $z$. The evanescent field significantly contributes to the detected signal only at $z<d / 2$.

However, there is a practical limitation on the GaAs layer thickness. The gating optical pulse can penetrate through the thin semiconductor layer (absorption coefficient of GaAs is $\sim 10^{4} \mathrm{~cm}^{-1}$ ) and reach the object through the aperture of the probe. This impact is undesirable, since it can change the optical properties of the object, for example, by generating photocarriers.

\section{B. Resolution}

Spatial resolution of the near-field probe is defined by the aperture size. To demonstrate it, we performed an edge resolution test on the probes with different aperture sizes. Boundary conditions for the electric field at a metallic edge are different for the two principal polarizations (parallel and perpendicular to the edge). If the edge is oriented parallel to the polarization of the incident terahertz pulse, then the electric field in the plane of the object exhibits a sharp contrast between the metallic and the open areas [18]. This sharp field contrast is used as a perfect edge object. The near-field image of it, therefore, gives an estimate of the spatial resolution of the probe.

The edge object is prepared by depositing a 600-nm gold film on one-half of a dielectric (GaAs) substrate. The edge is scanned over the probe at a distance $h \sim 2 \mu \mathrm{m}$. Fig. 4 shows the detected electric field amplitude as a function of the edge position with respect to the $5-\mu \mathrm{m}$ aperture probe $(L=4 \mu \mathrm{m})$. The electric field is measured at a fixed time delay of the gating optical pulse corresponding to the peak amplitude of the terahertz pulse as shown in the inset of the figure. The signal contrast is developed over a sample translation of $\sim 7 \mu \mathrm{m}$ (10-90\% criterion). The resolution test on the probes with larger apertures showed that spatial resolution scales with the aperture size.

The spatial resolution of the system is wavelength-independent. It is demonstrated by measuring the time-domain waveforms for every position of the edge with respect to the aperture. The spectral content of the field is obtained by applying the Fourier transform to the time-domain data. Fig. 5 shows the edge profile at various frequencies measured using the $10-\mu \mathrm{m}$ aperture probe $(L=4 \mu \mathrm{m})$. Identical resolution curves are obtained for a wide spectral window $(0.2-2.5 \mathrm{THz})$ limited only by the noise level.

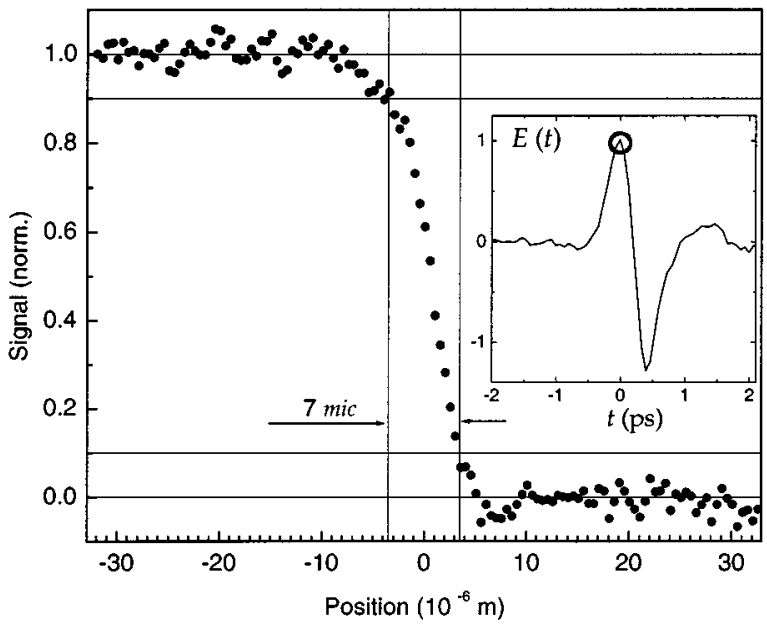

Fig. 4. Resolution test for the probe with $d=5 \mu \mathrm{m}$ and $L=4 \mu \mathrm{m}$. A circle in the inset shows the sampling time delay, at which the resolution curve is measured.

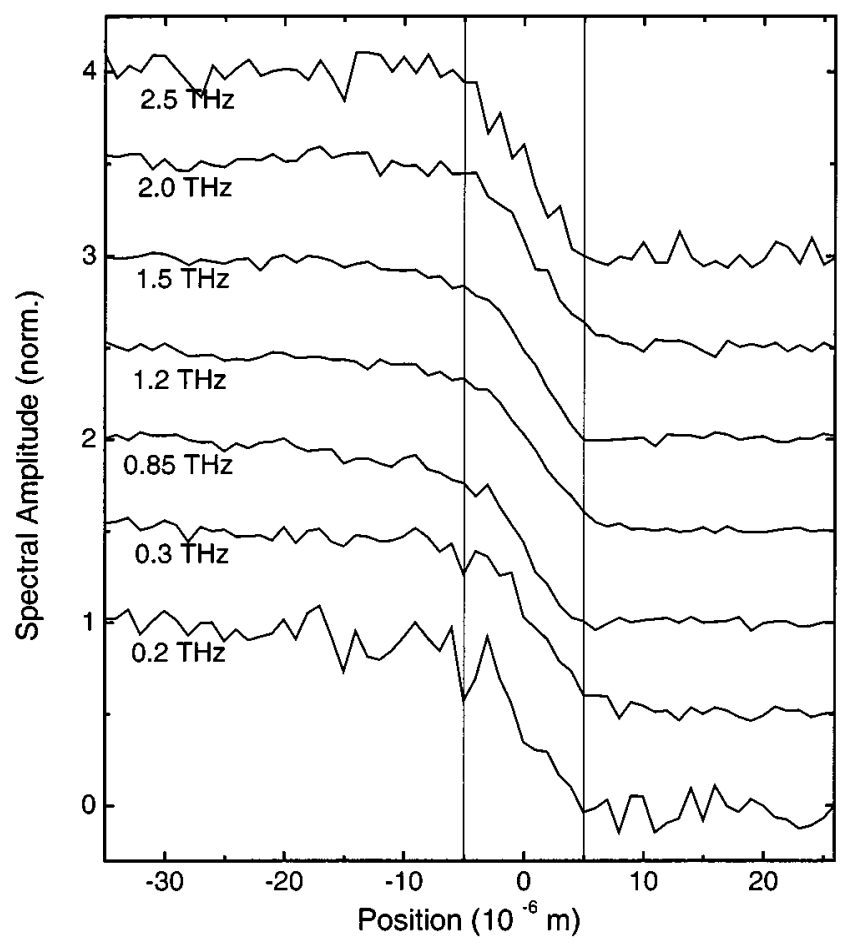

Fig. 5. Spatial resolution curves for different spectral components of the terahertz pulse measured using a near-field probe with $d=10 \mu \mathrm{m}$ and $L=4 \mu \mathrm{m}$.

\section{Aperture Size}

A limiting factor that prevents use of very small apertures for high resolution is the substantial reduction of the transmitted power as the aperture size decreases. According to the Bethe-Bouwkamp theory of transmission through a subwavelength aperture [16], [17], the electric field amplitude of the transmitted radiation decreases as the third power of the aperture size. The transmission coefficient is also frequency dependent, and, therefore, the near-field probe exhibits a nonuniform frequency response due to the aperture. 


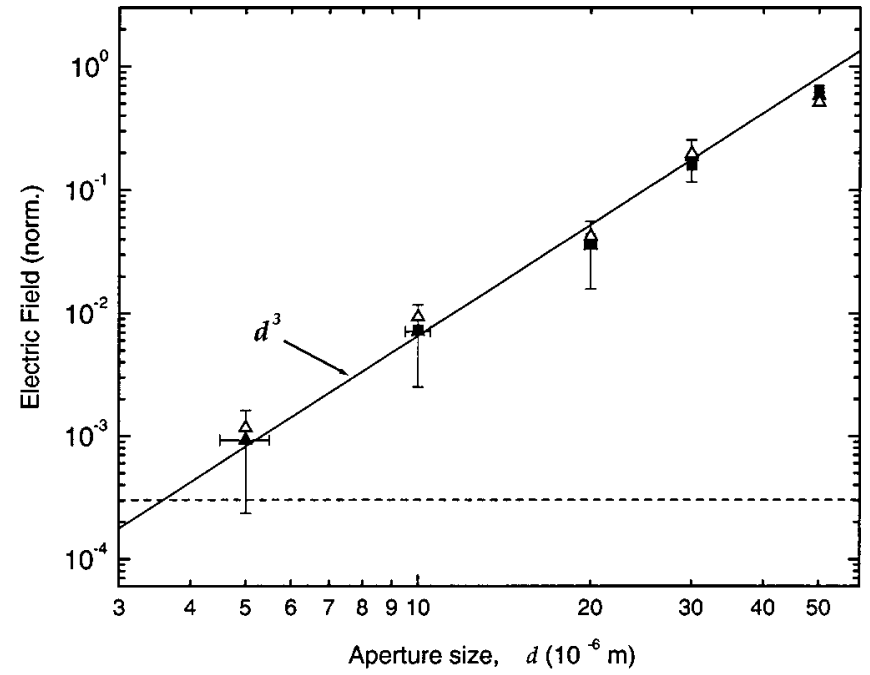

Fig. 6. Reduction of the probe aperture transmission. RMS values of the pulse waveforms are shown by solid symbols for two sets of tested probes. The peak amplitude of the pulses is shown by open symbols for comparison. The noise level is shown with a dashed line.

To study reduction of the transmitted field with the aperture size, we tested probes with apertures ranging from $d=50 \mu \mathrm{m}$ to $d=5 \mu \mathrm{m}$. All probes are fabricated with the GaAs layer of $\sim 4$ $\mu \mathrm{m}$. Due to temporal deformation of the pulses in the transmission process, attenuation is correctly represented in terms of the integrated intensity of the terahertz pulse $\left(\int|E(t)|^{2} d t\right)$ rather than the peak amplitude. The rms values of the measured waveforms are shown as solid symbols in Fig. 6. The measurements are in good agreement with the $d^{3}$ behavior. The peak amplitude of the detected terahertz pulse as a function of the aperture size is shown by open symbols for comparison. The electric field values are normalized in the same manner as in Fig. 3.

Below the aperature cutoff $(\omega<\pi / n d)$, the transmission coefficient of the aperture increases with frequency [16]. Therefore, the spectral content of the transmitted pulse shifts to higher frequencies compared to the incident pulse spectrum. Fig. 7(a) shows the normalized spectra of the incident terahertz pulse and the pulse transmitted through the 5- $\mu \mathrm{m}$ aperture probe with $L=4 \mu \mathrm{m}$ (solid curve). The incident pulse is measured using a detector fabricated in the same manner as the near-field probes, but without the metallic screen.

The spectrum effectively broadens for apertures smaller than $\sim 20 \mu \mathrm{m}$. The low-frequency part of the incident spectrum is suppressed by the aperture and the higher frequency components gain the relative weight in the pulse spectrum. For example, the spectrum of the pulse measured with the $5-\mu \mathrm{m}$ probe has its peak at $\sim 0.7 \mathrm{THz}$ and $\mathrm{FWHM}=1.1 \mathrm{THz}$, while the incident pulse with the peak at $0.52 \mathrm{THz}$ has the $\mathrm{FWHM}=0.7$ THz.

In the time domain, the spectral deformation corresponds to changes of the pulse duration and the oscillation period. For example, the pulse transmitted through the $50-\mu \mathrm{m}$ aperture contains $2-3$ cycles and extends in time to $\sim 2.1$ ps (FWHM), while the pulse transmitted through the $5-\mu \mathrm{m}$ aperture is almost single-cycle and lasts only 0.9 ps (FWHM). As shown in Fig. 7(b), the electric field of the pulse transmitted through the
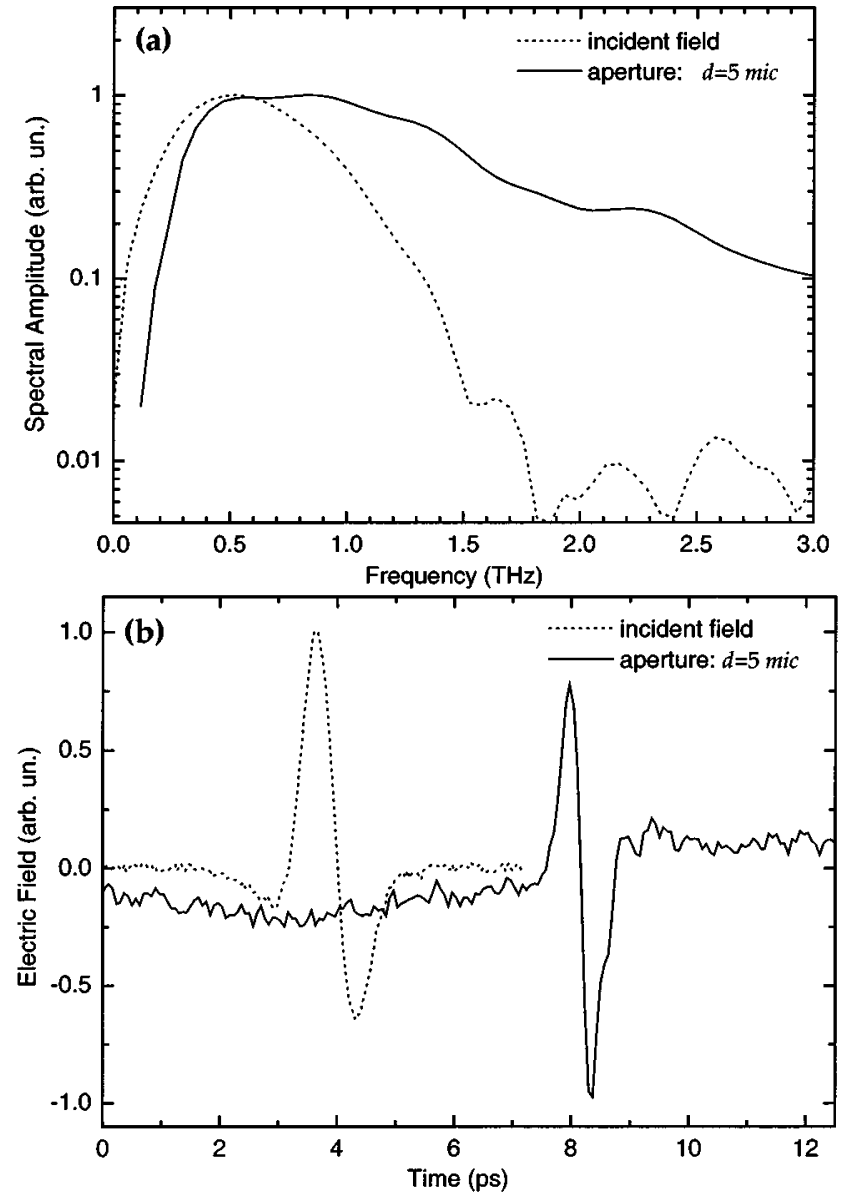

Fig. 7. (a) Normalized spectra of the incident terahertz pulse and the pulse detected by the $5-\mu \mathrm{m}$ probe $(L=4 \mu \mathrm{m})$. The spectrum of the pulse measured by the near-field probe is multiplied by $\sim 620$. (b) The corresponding electric field waveforms.

5- $\mu \mathrm{m}$ aperture also oscillates faster, compared to the incident pulse.

The weak transmission through a subwavelength aperture prevents the use of aperture sizes much smaller than $5 \mu \mathrm{m}$. The noise level (shown with the dotted line in Fig. 7) is 30 times lower than the signal amplitude. The $d^{3}$ line crosses the noise level at $d=3-4 \mu \mathrm{m}$. This establishes the potential limit of spatial resolution for our system. The amplitude of the signal is directly related to the amplitude of the incident pulse, and, in principle, a smaller aperture can be used with a more powerful source of terahertz pulses.

However, for a very small aperture, another factor should be considered. The metallic aperture screen allows a small fraction of the incident terahertz field to penetrate through the metal. This field does not contain the high spatial resolution information about the object, and, if it is comparable to the field coupled through the aperture, it would result in an image background. Therefore, the thickness of the metallic screen should be sufficient to attenuate the incident terahertz wave to a level lower than the signal transmitted through the aperture. In our case, the metallic screen is a $600-\mathrm{nm}$ gold film. The penetration depth of gold at $0.5 \mathrm{THz}$ is $\sim 50 \mathrm{~nm}$. It is experimentally estimated from the measurements of transmission through 50-, 100-, and 
200-nm-thick films on the GaAs surface. The measurements also showed that the gold film provides relatively uniform attenuation throughout the spectrum of the terahertz pulse. The total attenuation of the electric field due to the 600-nm film (including the surface reflections) is expected to be $\sim 10^{-5}$. Therefore, the $600-\mathrm{nm}$ gold screen is sufficient for near-field probes with apertures as small as $5 \mu \mathrm{m}$. However, the thickness must be increased if a smaller aperture is used.

It should be noted that the $d^{3}$ law is only valid for $d$ larger than the metallic screen thickness, but much smaller than the wavelength. Bethe derived this power law in the long wavelength approximation $(d \ll \lambda)$ for the infinitely thin metallic screen [17]. As the aperture size becomes comparable to the wavelength, the transmission coefficient saturates. We attribute deviation of the 50- $\mu \mathrm{m}$ aperture transmission from the power law to this saturation, rather than an experimental error. The spectrum of the incident terahertz pulse extends to the short wavelength region. Spectral components with wavelengths $\lambda<2$ nd propagate through the aperture without significant attenuation. The critical wavelength is $360 \mu \mathrm{m}$ for the $50-\mu \mathrm{m}$ aperture and 216 $\mu \mathrm{m}$ for the $30-\mu \mathrm{m}$ aperture. For very small apertures, comparable to the screen thickness, the power law is not expected to hold either, since the screen cannot be approximated as infinitely thin and additional attenuation in the aperture needs to be included.

\section{IMAGE PROPERTIES}

\section{A. Probe-Sample Separation}

The electric fields of high spatial frequency only exist in the proximity of the object (the evanescent fields) and decay over a distance comparable to the size of object features. To detect these fields, the near-field probe must be placed very close to the object. The aperture size resolution requires the probe-sample separation to be less than $d / 3$. The fast decay of the high spatial frequency fields is observed when performing the edge test for various separations between the probe and the object $h$. The sharp edge profile smears as $h$ increases.

Another effect related to the probe-sample separation is interference of waves reflected by the probe and the sample surfaces. This effect results in a variation of the detected field as a function of the probe-sample separation. The metallic surface of the near-field probe reflects the incident terahertz field toward the sample surface, at which the pulse partially reflects and eventually falls on the probe again. The time-delayed reflections are detected by the probe, and the delay corresponds to multiple double-paths between two surfaces. If the probe-sample separation is larger than half of the terahertz pulse length in free space, then the reflections can be easily distinguished in the time domain. As the probe-sample separation decreases, the reflections start overlapping temporally. When the sample probe separation is smaller than $\sim 15 \mu \mathrm{m}$ (the delay between reflections is $<0.1$ ps), the reflections overlap constructively and the waveform is almost indistinguishable from that of the incident pulse, but the waveform amplitude increases as distance $h$ decreases. This effect is not related to the evanescent field and is completely described by the interference. An example of the approach curve

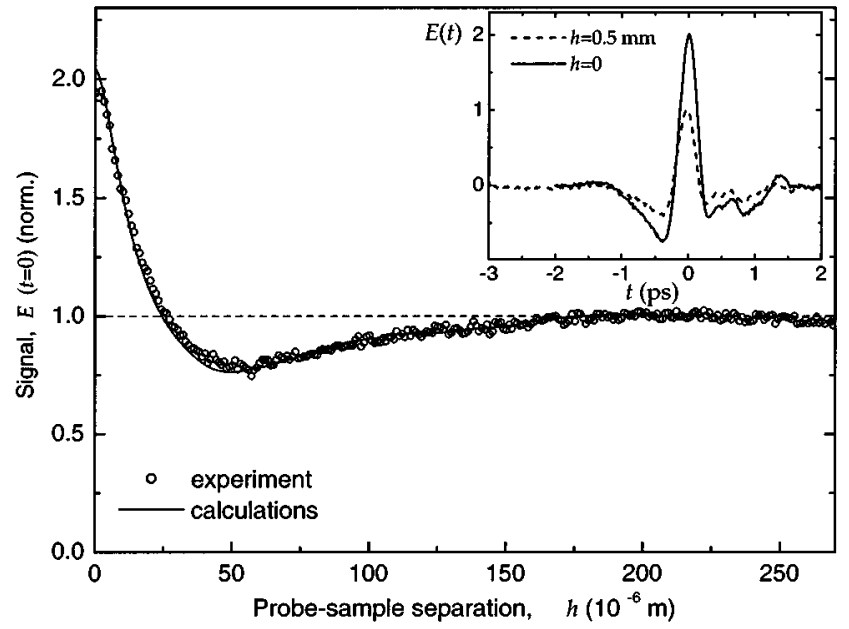

Fig. 8. Measured (circles) and calculated (solid line) approach curves for the $10-\mu \mathrm{m}$ probe $(L=4 \mu \mathrm{m})$. The inset presents the time-domain waveforms measured at $h=0$ and $h=500 \mu \mathrm{m}$.

measured when the time delay is fixed at the peak of the terahertz pulse is shown in Fig. 8. The experiment is performed using the $10-\mu \mathrm{m}$ aperture probe $(L=4 \mu \mathrm{m})$. A sapphire plate is chosen as the sample for the high dielectric constant. The solid line shows an approach curve calculated using the waveform of the incident terahertz pulse and the interference model. The calculations and the experimental data are in excellent agreement. We can emphasize two properties of the approach curve: the shape depends on the terahertz pulse waveform and the amplitude depends on the dielectric constant of the sample. Note that the variation of the signal is substantial due to the high reflectivity of the probe and sample surfaces.

In practical terahertz near-field imaging, the probe-sample separation is less than several micrometers. The waveform distortion due to interference is negligible at this range. However, the variation of the amplitude of the detected terahertz field can create an uneven background in the image if the separation is not maintained constant during the scan.

\section{B. Evolution of Near-Field Images}

The THz-TDS technique allows studying objects in the time domain by sampling the electric field of the terahertz pulse using short optical pulses. Contrary to continuous wave illumination, when an image pattern (which corresponds to the optical intensity) is stationary, images recorded using the THz-TDS system evolve in time. Object features respond to the electromagnetic field of the incident terahertz pulse differently depending on their shape, optical properties, and orientation.

In the series of images in Fig. 9, we demonstrate the change of the electric field pattern with time. An object in this experiment is a gold dipole antenna embedded in a planar transmission line. A schematic diagram of the sample is shown in Fig. 9(a). The antenna is lithographically printed on a sapphire substrate and consists of two sharp-ended 50- $\mu \mathrm{m}$-long arms, slightly shifted with respect to each other. The $20-\mu \mathrm{m}$-wide striplines are separated by $105 \mu \mathrm{m}$. The dipole arms are oriented parallel to the direction of polarization. The near-field images are obtained at 


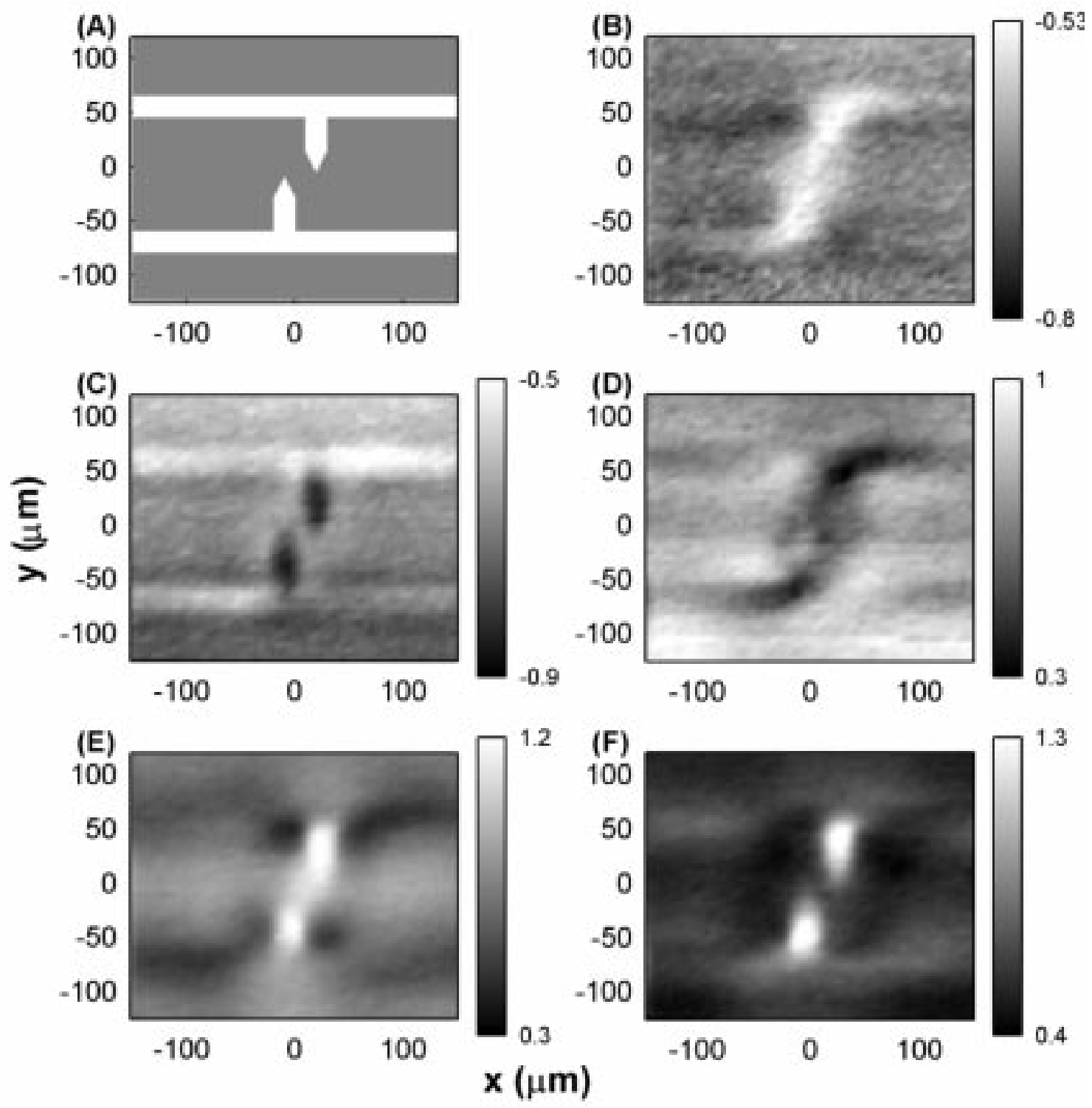

Fig. 9. Series of near-field images of electric field (b-f) in the plane of a planar antenna on sapphire (a). The images are taken at different time delays. The gray level corresponds to the measured electric field, normalized to the pulse peak amplitude.

consequent moments in time using the $10-\mu \mathrm{m}$ aperture probe $(L=4 \mu \mathrm{m})$.

Frames $b$ and $d$ (measured at $t=-0.5$ ps and $t=0$, respectively) correspond to the negative and positive peaks of the terahertz pulse waveform. The images are similar except for polarity. The dipole creates a shadow in the center, which extends slightly toward the striplines. The antenna pattern is barely recognizable in these images.

All object features develop well in frame $c(t=-0.33 \mathrm{ps})$, which corresponds to a moment between the negative and positive peaks of the terahertz pulse. Both the striplines and the dipole arms are clearly seen. However, the object parts oriented perpendicularly to the polarization direction appear brighter, and the parts oriented parallel to it appear darker with a higher contrast. Resolution is comparable in the both directions.

Frames $e(t=0.17 \mathrm{ps})$ and $f(t=0.33 \mathrm{ps})$ show another effect. The dipole arms are imaged as bright spots with high con- trast. Both images correspond to moments when the peak of the terahertz pulse has already passed. However, the measurements show high electric field in the dipole region.

It is informative to consider the whole time-domain waveforms measured in various points of the object. The waveforms are quite different in the dipole region. They exhibit larger amplitude and temporal delay compared to the waveforms measured at a distance (150-200 $\mu \mathrm{m})$ aside from the antenna. As a result, the dipole appears glowing after the field of the incident pulse has decreased ( $t \sim 0.2-0.5 \mathrm{ps})$. In addition, the temporal evolution of the field changes. It implies that the dipole, as a resonant structure, locally perturbs the incident terahertz field.

It should be mentioned that an image in collection mode is not a direct replica of the instantaneous electric field scattered by the object. The probe aperture alters the detected waveform. In principle, the original waveform can be extracted if the transfer function of the aperture is known. Despite the complex interaction 
between the object and the probe, Fig. 9 shows that the near-field technique allows high spatial resolution imaging using terahertz waves.

\section{CONCLUSION}

We demonstrate that the terahertz near-field scanning imaging technique is capable of sub-10- $\mu \mathrm{m}$ spatial resolution. Significant improvement of resolution over the diffraction limit is possible owing to the efficient near-field probe design and the exceptional sensitivity of the THz-TDS technique. Spatial resolution of $7 \mu \mathrm{m}$ is experimentally demonstrated for broadband terahertz pulses (120-1500 $\mu \mathrm{m})$. The present studies consider only the collection-mode setup; however, the near-field probe can be used in illumination mode as well [10].

Using the time-resolved measurements, two types of terahertz near-field images can be constructed:

1) time-domain frames of electric field;

2) chromatic images (amplitude and phase).

The latter method opens the possibility for near-field spectroscopy at terahertz frequencies. The image acquisition time in this case increases considerably, since a complete time-domain waveform is measured for every pixel.

Near-field image formation and effects of interaction between the probe and objects still need to be studied in order to transfer this technique into a reliable characterization tool. In this respect, we would like to mention the finite-difference time-domain numerical method [12], [19], [20], which can be used to simulate terahertz near-field images.

\section{REFERENCES}

[1] D. M. Mittleman, M. Gupta, R. Neelamani, R. G. Baraniuk, J. V. Rudd, and M. Koch, "Recent advances in terahertz imaging," Appl. Phys. B, vol. 68, pp. 1085-1094, 1999.

[2] M. C. Nuss and J. Orenstein, "Terahertz time-domain spectroscopy," in Millimeter-Wave Spectroscopy of Solid. ser. Springer Topics in Applied Physics, G. Gruner, Ed. Berlin, Germany: Springer, 1998, vol. 74.

[3] M. van Exter and D. R. Grischkowsky, "Characterization of an optoelectronic terahertz beam system," IEEE Trans. Microwave Theory Tech., vol. 38, pp. 1684-1691, 1990.

[4] B. B. Hu and M. C. Nuss, "Imaging with terahertz waves," Opt. Lett., vol. 20, pp. 1716-1719, 1995.

[5] P. Y. Han, G. C. Cho, and X.-C. Zhang, "Time-domain transillumination of biological tissues with terahertz pulses," Opt. Lett., vol. 25, pp. 242-244, 2000

[6] S. Mickan, D. Abbott, J. Munch, X.-C. Zhang, and T. Van Doorn, "Analysis of system trade-offs for terahertz imaging," Microelectron. J., vol. 31, pp. 503-514, 2000.

[7] S. Hunsche, M. Koch, I. Brener, and M. C. Nuss, "THz near-field imaging," Opt. Commun., vol. 150, pp. 22-26, 1998.

[8] K. Wynne and D. A. Jaroszynski, "Superluminal terahertz pulses," Opt. Lett., vol. 24, pp. 25-27, 1998.

[9] O. Mitrofanov, I. Brener, R. Harel, J. D. Wynn, L. N. Pfeiffer, K. W. West, and J. Federici, "Terahertz near-field microscopy based on a collection mode detector," Appl. Phys. Lett., vol. 77, pp. 3496-3498, 2000.

[10] O. Mitrofanov, I. Brener, M. C. Wanke, R. R. Ruel, J. D. Wynn, A. J. Bruce, and J. Federici, "Near-field microscope probe for far infrared time domain measurements," Appl. Phys. Lett., vol. 77, pp. 591-593, 2000.

[11] Q. Chen, Z. Jiang, G. X. Xu, and X.-C. Zhang, "Near-field terahertz imaging with a dynamic aperture," Opt. Lett., vol. 25, pp. 1122-1124, 2000 .

[12] O. Mitrofanov, R. Harel, M. Lee, L. N. Pfeiffer, K. West, J. D. Wynn, and J. Federici, "Study of single-cycle pulse propagation inside a $\mathrm{THz}$ near-field probe," Appl. Phys. Lett., vol. 78, pp. 252-254, 2001.
[13] K. Wynne, J. J. Carey, J. Zawadzka, and D. A. Jaroszynski, "Tunneling of single-cycle terahertz pulses through waveguides," Opt. Commun., vol. 176, pp. 429-435, 2000.

[14] R. D. Grober, T. Rutherford, and T. D. Harris, "A modal approximation for the electromagnetic field of a near-field optical probe," Appl. Opt., vol. 35, pp. 3488-3495, 1996.

[15] Y. Leviatan, "Study of near-zone fields of a small aperture," J. Appl. Phys., vol. 60, pp. 1577-1583, 1986.

[16] H. A. Bethe, "Theory of diffraction by small holes," Phys. Rev., vol. 66, pp. 163-182, 1944.

[17] C. J. Bouwkamp, "On Bethe's theory of diffraction by small holes," Philips Res. Rep., vol. 5, pp. 321-332, 1950.

[18] I. Brener, S. Hunsche, Y. Cai, M. C. Nuss, J. Wynn, J. Lopata, and L. Pfeiffer, "Time resolved near field imaging and diffraction with subwavelength far-infrared dipole sources," in Ultrafast Phenomena XI, 1998, pp. 171-172.

[19] A. Taflove and S. Hagness, Computational Electrodynamics: The Finite-Difference Time-Domain Method. Norwood, MA: Artech House, 2000

[20] J. Bromage, S. Radic, G. P. Agrawal, C. R. Stroud, Jr., P. M. Fauchet, and R. Sobolevski, "Spatiotemporal shaping of half-cycle terahertz pulses by diffraction through conductive apertures of finite thickness," J. Opt. Soc. Amer. B, vol. 15, pp. 1399-1405, 1998.

Oleg Mitrofanov received the B.Sc. degree in physics from Moscow State University, Russia, in 1997 and the Ph.D. degree in applied physics from the New Jersey Institute of Technology, Newark, in 2001.

He joined Bell Laboratories-Lucent Technologies, Murray Hill, NJ, in 1998, working on spectroscopy and near-field imaging with terahertz pulses. He is currently a Member of Technical Staff at Bell Laboratories, Murray Hill.

Mark Lee is a Member of Technical Staff at Bell Laboratories-Lucent Technologies, Murray Hill, NJ. Previously, he was a Professor of physics at the University of Virginia, Richmond. His research interests have included quantum correlated systems and millimeter-wave electrodynamic properties of superconductors, semiconductors, and polymers.

Julia W. P. Hsu received the B.S.E. degree in chemical engineering from Princeton University, Princeton, NJ, in 1985 and the M.S. and Ph.D. degrees in physics from Stanford University, Stanford, CA, in 1987 and 1991, respectively.

She is a Member of Technical Staff at Bell Laboratories, Lucent Technologies, Murray Hill, NJ. From 1993 to 1999, she was an Associate Professor and an Assistant Professor of physics at the University of Virginia, Richmond. Her research focuses on local characterization of electronic and photonic materials and devices using scanning probe techniques.

Dr. Hsu is a member of the Materials Research Society and the American Physical Society. She received the American Physical Society Apker Award (1986), the National Science Foundation Young Investigator Award (1993), and the Sloan Foundation Research Fellowship (1994).

Igal Brener (M'99) received the B.Sc. degree in electrical engineering, the B.A. degree in physics, and the Ph.D. degree in physics from the Technion-Israel Institute of Technology, Haifa, in 1983, 1983, and 1991, respectively.

He was with National Semiconductors working in microprocessor VLSI design from 1983 to 1986. In 1991, he joined Bell Laboratories, Holmdel, NJ, as a Postdoctoral Member of Technical Staff to work in ultrafst measurements of semiconductor quantum wells and terahertz emission and coherent phenomena in semiconductors. He was a Member of Technical Staff at the Optics Physics Research Department, Bell Laboratories, Murray Hill, NJ, from 1993 to 2000. His research dealt with wavelength conversion and novel nonlinear-optical devices for optical communication, near-field imaging in the far-infrared and coherent phenomena in semiconductor microcavaties. He joined Tellium Inc., Oceanport, NJ, in 2000, where he works in optical switching. He has authored contributions to more than 60 publications and has received five patents.

Dr. Brener is a member of the Optical Society of America and the IEEE Laser and Electro-Optics Society. 
Roey Harel received the B.Sc. degree in physics from Tel-Aviv University, Israel, in 1989. He received the M.Sc. degree in physics and the Ph.D. degree from the Technion-Israel Institute of Technology, Haifa, in 1996 and 2000, respectively.

He worked in Rafael, Israel Armament Development Authority, from 1989 to 1994. His Ph.D. research was on the coherent properties of semiconductor micorcavaties. In 1999, he joined Bell Laboratories, Murray Hill, NJ, as a Research Student, where he became a Member of Technical Staff in 2000. His research dealt with periodically poled $\mathrm{LiNbO} 3$ waveguide wavelength converters. Currently, he is with Tellium, Inc., Oceanport, NJ, where he works on all-optical switching.

Dr. Harel is a member of the Optical Society of America.

John F. Federici received the B.S. degree in physics from the University of Notre Dame, Notre Dame, IN, in 1983 and the Ph.D. degree from Princeton University, Princeton, NJ.

He joined Bell Laboratories-Lucent Technologies in 1988, first in a postdoctoral position and then as a Member of Technical Staff. He joined the physics Faculty of the New Jersey Institute of Technology, Newark, in 1992. He is currently the Associate Chair of the Department of Physics and a Faculty Member of the Department of Biomedical Engineering. His current research interest include the study of condensed matter and biological tissues using optical techniques such as polarization imaging, far-infrared (terahertz) pump/probe spectroscopy, photoconductivity, photoluminescence, and thermal modulation spectroscopy.
James D. Wynn is the Process Development Manager at Priceton Optronics, Inc., in Mercerville, NJ. Previously, he was a Member of Technical Staff at Bel Laboratories, Murray Hill, NJ.

Loren N. Pfeiffer, photograph and biography not available at the time of publication.

Ken W. West, photograph and biography not available at the time of publication. 